\title{
Effects of Prenatal Dexamethasone or Terbutaline Exposure on Development of Neural and Intrinsic Control of Heart Rate
}

\author{
QING-CHANG HOU AND THEODORE A. SLOTKIN
}

Department of Pharmacology. Duke University Medical Center, Durham, North Carolina 27710

\begin{abstract}
This study compares the effects of prenatal exposure to terbutaline (a $\beta$-adrenergic agonist) and dexamethasone (a glucocorticoid) on the development of heart rate control mechanisms in the rat. Both drugs produced a persistent reduction in resting heart rate appearing during the 2nd postmatal wk, but by different mechanisms. Terbutaline affected the development of autonomic input from the CNS, characterized by a premature shift from sympathetic to parasympathetic dominance; thus, heart rate differences between terbutaline-exposed animals and controls resolved with acute treatment with a ganglionic blocking agent (chlorisondamine). Dexamethasone did not alter neural input to the myocardium (its actions were not reversed by ganglionic blockade), but instead reduced the intrinsic heart rate; the prenatal glucocorticoid treatment also reduced the sensitivity of the myocardium to $\beta$-adrenergic stimulation, a factor that could contribute to the alterations in intrinsic rate. These results suggest the potential need for studies of the functional cardiovascular consequences of fetal or neonatal therapeutic interventions with glucocorticoids or adrenergic agents. (Pediatr Res 26: 554-557, 1989)
\end{abstract}

During critical periods, the developing nervous system undergoes "programing" of its future responses to external stimuli and is thus particularly vulnerable to neuroactive substances. Numerous studies have shown long-lasting or permanent alterations in neurochemical and neurobehavioral function as a result of prenatal or neonatal drug treatments acting to stimulate or block transmitter receptor sites. Indeed, the development of receptor sites and their ability to elicit target organ responses appear to be directly dependent on exposure of the sites to transmitter at an approptiate maturational phase (1-4). In the peripheral autonomic system of the neonatal rat, we have found that early postnatal $\beta$-adrenergic input is required for sympathetic target organs to develop appropriate responsiveness to neural and hormonal input (5-9), and that disruption of neonatal neural or hormonal signals can effect changes in heart growth and heart rate lasting into adulthood $(10-13)$.

Our study extends these findings to the prenatal period by examining the consequences of late gestational exposure to dexamethasone (a glucocorticoid) or terbutaline (a $\beta$-adrenergic agonist), representatives of agents used in human prematurity $(14,15)$ to promote fetal/neonatal lung development (both drugs) and to arrest labor ( $\beta$-agonists); the use of either drug in the management of maternal asthma can also result in prenatal

Recejved May 1, 1989; accepted July 26, 1989

Correspondence Dr. T. A. Slotkin, Box 3813, Duke University Medical Center, Durham, NC 27710.

Supported by USPHS HD-09713 and EPA CR-813769. exposure. Actions of glucocorticoids and $\beta$-agonists directed toward adrenergic function are dissimilar: dexamethasone affects coupling of $\beta$-receptors to cellular function $(16,17)$, whereas terbutaline directly stimulates the receptor site, thus simulating actions of the natural neurotransmitter, norepinephrine (18). Accordingly, the results presented here address the question of whether intrinsic or neural control of heart rate in the developing rat are affected by prenatal exposure to either agent, using dose regimens of terbutaline and dexamethasone previously found to produce stimulation of lung function $(14,15,19,20)$.

\section{MATERIALS AND METHODS}

Timed pregnant Sprague Dawley rats (Zivic-Miller Laboratories, Allison Park, PA) were shipped on the 4th to 7 th $d$ of gestation, were housed individually and were given food and water ad libitum. On gestational d 17,18, and 19, dams were given either $0.8 \mathrm{mg} / \mathrm{kg}$ subcutaneously of dexamethasone phosphate (Merck Sharp and Dohme, Rahway, NJ) or $10 \mathrm{mg} / \mathrm{kg}$ of terbutaline sulfate (Geigy Pharmaceuticals, Ardsley, NY); controls received an equivalent volume of saline vehicle. After birth, pups were randomized within their respective treatment groups and redistributed to the nursing dams with litter size maintained at 10-12 pups; randomization was carried out again just before each experiment and in addition, pups were selected from different cages and sex-matched between treatment groups using approximately equal proportions of males and females.

Rats were anesthetized with urethane $(1.2 \mathrm{mg} / \mathrm{kg}$ intraperitoneally; Sigma Chemical Co., St. Louis, MO) and body temperature was maintained at $37^{\circ} \mathrm{C}$ with a rectal thermistor and infrared heating lamp. Needle electrodes were inserted in to the limbs and heart rate was determined by counting QRS complexes in a 5-s period using EKG lead II. Temperature was allowed to stabilize and heart rate measurements taken every 1 to $2 \mathrm{~min}$. When a constant baseline value was obtained, the rate was recorded as the "resting" heart rate. The animals were then given chlorisondamine chloride $(2.5 \mathrm{mg} / \mathrm{kg}$ subcutaneously; Ciba Pharmaceuticals, Summit, NJ) to block autonomic ganglia and adrenomedullary catecholamine secretion, and thus to prevent participation of reflex neural signals in heart rate regulation $(21,22)$. The heart rate was allowed to stabilize to establish the "basal" heart rate (i.e. the intrinsic rate without neural input); the acute change caused by chlorisondamine (the difference between resting and basal heart rate) also provides a measure of the relative balance between sympathetic and parasympathetic contributions to resting heart rate (22). Subsequent to the chlorisondamine treatment, a submaximally-effective test dose of $d, l$-isoproterenol sulfate $(1.5 \mu \mathrm{g} / \mathrm{kg}$ subcutaneously; Sigma) was given and the peak effect on heart rate recorded. Finally, the "maximal" heart rate was established by giving a high dose of isoproterenol $(50 \mu \mathrm{g} / \mathrm{kg})$, which was repeated once or twice at short time intervals until no further increase could be obtained. Injection of equivalent vol- 
umes of the $0.9 \%$ saline vehicle used for these test drugs had no effect on heart rate (data not shown).

Statistics. Data are presented as means and standard errors, with statistical comparisons made by analysis of variance, with factors of age and prenatal dexamethasone or terbutaline treatment; as no interactions of treatment $x$ sex were found (data not shown), results for males and females were combined. Significance was assumed at a criterion of $p<0.05$ for main treatment effects and at $p<0.10$ for interaction of the two variables (23). POSt hoc analysis of individual age points at which the prenatally treated groups differed from control (Duncan's multiple range test) was conducted only where a significant treatment $x$ age interaction was obtained. Each age point examined in this study was conducted with separate groups of animals (i.e. no repeated measurements across age); in each treatment group, eight animals were studied at $1 \mathrm{~d}$ of age, eight at $2 \mathrm{~d}, 16$ at 5-6 d, 16 at 9-10 $\mathrm{d}, 16$ at $16-17 \mathrm{~d}, 14$ at $23-24 \mathrm{~d}, 13$ at $31-32 \mathrm{~d}$, eight at $37-38 \mathrm{~d}$ and 13 at $48-49 \mathrm{~d}$.

\section{RESULTS}

Administration of dexamethasone on gestational d 17, 18, and 19 had no effect on maternal viability or on the proportion of dams giving birth, but did cause a slight lowering (ca. 10\%) of litter size and $15 \%$ mortality in the first $10 \mathrm{~d}$ (data not shown). After birth, animals in the dexamethasone group showed a persistent slowing of wt gains, which did not resolve even in young aduithood (Fig. 1). In contrast, prenatal terbutaline exposure had no effect on birth or viability characteristics (data not shown) and had only small, short-term effects on postnatal growth (Fig. 1).

In control rats, resting heart rate was low in the immediate postnatal period, rose to a peak during the 2 nd and 3 rd wk after birth and declined slightly thereafter (Fig. 2). Although animals exposed to dexamethasone or terbutaline also displayed the same basic development pattern, both treatment groups exhibited persistent deficits in resting rate beginning during the 2 nd wk.

To distinguish whether these differences reflected altered autonomic input to the heart or rather intrinsic rate changes, we examined basal heart rates after interruption of neural input by chlorisondamine (Fig. 3, top). In control rats, the developmental rise of basal heart rate occurred during the second to 3 rd postnatal

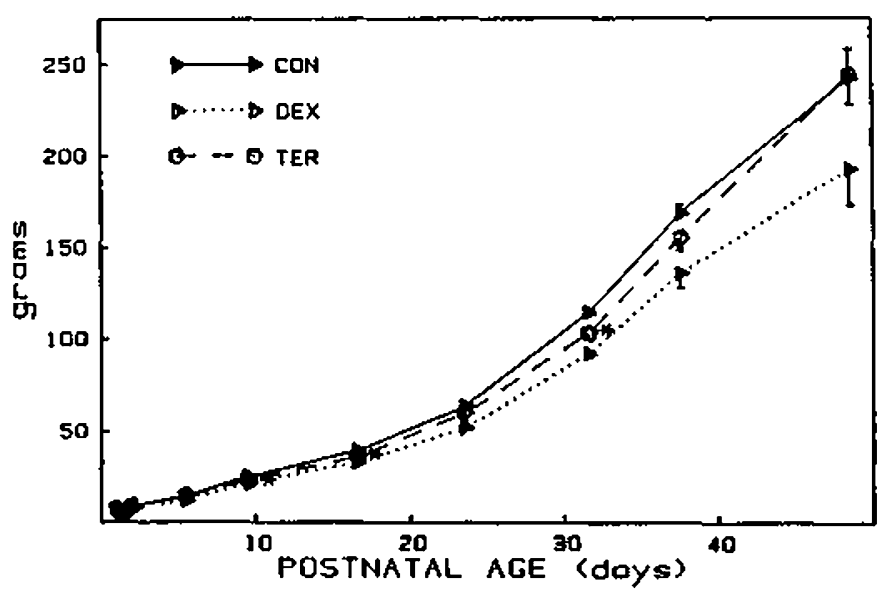

Fig. 1. Effects of prenatal dexamethasone $(D E X)$ or terbutaline (TER) treatment on body wt compared to controls (CON). Data represent means and SE of values from eight to 16 rats in each group at each age. Analysis of variance (performed on log-transformed data because of heterogeneous variance) indicates a significant increase in wt with age (main effect of age) and an overall decrease in body wt gains caused by dexamethasone (main treatment effect). Terbutaline exposure also attenuated wt gains in an age-dependent manner (main treatment effect and interaction of treatment $\times$ age) and asterisks denote individual ages at which the terbutaline group differs significantly from control.

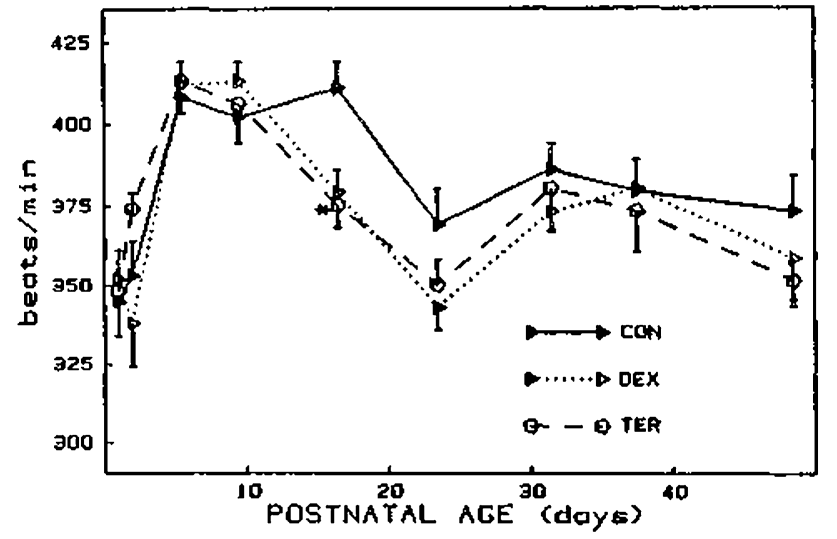

Fig. 2. Effects of prenatal dexamethasone $(D E X)$ or terbutaline $(T E R)$ treatment on resting heart rate compared to controls (CON). Data represent means and SE of values from eight to 16 rats in each group at each age. Analysis of variance indicates a significant change in rate with age (main effect of age) and an overall attenuation caused by dexamethasone (main treatment effect). Terbutaline caused an agedependent alteration (significant interaction of treatment $\times$ age; asterisk denotes individually significant value).

wk and, unlike resting rate, showed no subsequent fall-off. Animals exposed to prenatal dexamethasone displayed the same eventual deficit in basal heart rate as for resting rate; however, in the terbutaline group, chlorisondamine eliminated most of the differences that had been seen in resting rate. Because chlorisondamine interrupts all autonomic transmission, the acute change in heart rate caused by this drug gives an estimate of the relative balance of parasympathetic versus sympathetic contributions to resting heart rate: a fall in rate after chlorisondamine indicates sympathetic dominance, whereas a rise indicates parasympathetic dominance. Chlorisondamine evoked only small changes in rate in the immediate postnatal period in control rats, but a large decrease was obtained toward the end of the 1st wk and into the 2 nd wk (Fig. 3, bottom); by weaning ( $22 \mathrm{~d}$ ), acute chlorisondamine caused an increase in rate instead of a decrease. Although prenatal exposure to dexamethasone had some small initial effects, it did not change the general characteristics of this pattern. However, terbutaline promoted the developmental switchover to the phase in which heart rate increases after chlorisondamine, with both an earlier appearance of this phase and a promotion of the peak of activity on days $23-24$.

Postsynaptic sensitivity of heart rate to $\beta$-adrenergic stimulation, evaluated by acute challenge with a submaximally effective dose of isoproterenol, also showed developmental increases in control rats (Fig. 4). Dexamethasone had a small, but statistically significant, decremental effect on this response, but terbutaline did not. Supramaximally effective doses of isoproterenol, used to evaluate maximal heart rate, showed much greater developmental rises in effect over this period (Fig. 5). Again, dexamethasone, but not terbutaline, significantly reduced this response by small amount; because both the basal rate and maximal rate were lowered in the dexamethasone group, evaluation of the maximal rate as the increase over basal showed an even smaller difference from control values (data not shown).

\section{DISCUSSION}

Our results indicate that prenatal exposure to either dexamethasone or terbutaline interferes with the development of heart rate control mechanisms, exemplified by a prolonged decrease in resting heart rate beginning at the end of the 2 nd postnatal wk. The timing with which this alteration occurred suggested that we should examine whether effects on neural input could participate in some of the actions. Autonomic efferent control of heart rate develops from the end of the 1st postnatal wk to the 3 rd wk in 